\title{
Behavior of Friedmann-Robertson-Walker Cosmological Models in Scalar-Tensor Gravity
}

\author{
Shawn J. Kolitch * \\ Department of Physics \\ University of California \\ Santa Barbara, CA 93106-9530 \\ Douglas M. EARDLEy ${ }^{\dagger}$ \\ Institute for Theoretical Physics \\ University of California \\ Santa Barbara, CA 93106-4030 \\ (June , 1994)
}

\begin{abstract}
We analyze solutions to Friedmann-Robertson-Walker cosmologies in BransDicke theory, where a scalar field is coupled to gravity. Matter is modelled by a $\gamma$-law perfect fluid, including false-vacuum energy as a special case. Through a change of variables, we reduce the field equations from fourth order to second order, and they become equivalent to a two-dimensional dynamical system. We then analyze the entire solution space of this dynamical system, and find that many qualitative features of these cosmologies can be gleaned, including standard non-inflationary or extended inflationary expansion, but also including bifurcations of stable or unstable expansion or contraction, noninflationary vacuum-energy dominated models, and several varieties of "coasting," "bouncing," "hesitating," and "vacillating" universes. It is shown that inflationary dogma, which states that a universe with curvature and dominated by inflationary matter will always approach a corresponding flatspace solution at late times, does not hold in general for the scalar-tensor theory, but rather that the occurence of inflation depends upon the initial energy of the scalar field relative to the expansion rate. In the case of flat space $(k=0)$, the dynamical system formalism generates some previously
\end{abstract}

*E-Mail address: kolitch@nsfitp.itp.ucsb.edu

$\dagger$ E-Mail address: doug@sbitp.ucsb.edu 
known exact power-law solutions.

04.50.+h, 98.80.Hw, 02.30.Hq

Typeset using REVTEX 


\section{INTRODUCTION}

It was first recognized by Guth that inflation, i.e., a period of superluminal expansion in the early universe, solves many of the problems of the standard model of cosmology, a.k.a. the "hot big bang" theory [1]. These include the smoothness/horizon problem, the flatness/age problem, the structure problem and the massive relics problem. (A review of these problems, and their resolution in an inflationary scenario, is given in [2].) However, in the original scenario proposed by Guth, one finds that the deSitter expansion induced by vacuum energy density (or, equivalently, by a cosmological constant) in ordinary general relativity is impossible to exit from via barrier penetration in a way that permits sufficient inflation to solve the aforementioned problems, while still reheating the universe afterwards [3]. In particular, it can be shown that if the tunneling transition rate for the inflaton field is small enough to allow for sufficient inflation, then it is too small to nucleate truevacuum bubbles fast enough to collide and reheat the universe, and the phase transition to a radiation-dominated era is never completed. A number of possible solutions have been proposed for this "graceful exit problem," including an extremely flat inflation potential ("new inflation"), so that inflation occurs as the field "rolls down" towards its true-vacuum state, without any barrier penetration being necessary [4]; and including "chaotic inflation," in which only certain regions of the universe inflate to large size before the true vacuum is restored [5].

One such proposal, "extended inflation" [6], based on the Brans-Dicke (BD) theory of gravity [7], solves the graceful exit problem while still allowing the phase transition that ends inflation to be completed via bubble nucleation, by employing power-law rather than exponential expansion. This permits eventual complete reheating even if the tunneling transition rate for the inflaton is small enough to ensure that inflation continues at least until the relevant cosmological problems are solved.

In its original form, this proposal fails due to the creation of unacceptably large microwave background perturbations by collisions between big bubbles [8]. To avoid this "big bubble problem", the coupling constant must take on a value in the range $\omega \leq 25$ during inflation, in conflict with limits set by recent experiments of $\omega>500$ [9]. This conflict may be circumvented by further alterations of the gravitational sector, such as a potential for the BD scalar [10]. In addition to the big bubble problem, however, one finds that the spectrum of density perturbations produced by quantum fluctuations of the BD scalar during inflation is distorted away from scale-invariance, so that COBE measurements of the spectral index of the perturbations put a further constraint on the allowed range of $\omega$ during inflation. Taken together, these constraints appear to rule out many extended inflation models [11. Models which survive include those with a variable coupling constant such as hyperextended inflation [12], and hybrid models which include both a first-order phase transition and a period of slow-roll [13].

Recent interest in BD theory is also motivated by its relation to low-energy bosonic string theory. We can write the action for BD theory as

$$
S_{B D}=\int d^{4} x \sqrt{-g}\left(-\phi R+\omega \frac{\phi^{, \mu} \phi_{, \mu}}{\phi}+16 \pi \mathcal{L}_{m}\right),
$$

where $R$ is the scalar curvature, $\omega$ is the dimensionless BD coupling constant, and $\phi$ is the 
BD scalar field. The low-energy effective action for bosonic string theory can be written in the form [14

$$
S_{e f f}=\int d^{4} x \sqrt{-g} e^{-2 \Phi}\left(R+4 \Phi^{, \mu} \Phi_{, \mu}-\frac{1}{12} H^{2}\right) .
$$

Here $R$ is again the scalar curvature, but now $\Phi$ is the dilaton field and $H^{2} \equiv H_{\mu \nu \rho} H^{\mu \nu \rho}$, where $H_{\mu \nu \rho}$ is the totally antisymmetric three-form field. If we make the substitution $\phi=$ $e^{-2 \Phi}$ we see that, considering only the scalar and tensor components and neglecting the matter, the two theories are identical in the low-energy limit if $\omega=-1$. It should be emphasized, however, that the two theories differ in their couplings of the scalar field to the other matter. The renewed interest in scalar-tensor gravitation has led to several recent investigations into the generation of exact solutions for cosmology in such theories [30], as well as to some qualitative studies of the models which result [31,32].

There is considerable interest, therefore, both from inflationary cosmology and from string theory, to revisit cosmology in the scalar-tensor gravity of Brans and Dicke. Many papers were of course written on BD cosmology in the 1960's and 1970's 15 19]; interest then declined, partly because observational tests of gravity put increasingly stringent lower limits on the $\mathrm{BD} \omega$ in the present universe, and partly because fundamental scalar fields fell out of theoretical favor. However, string theory restored the theoretical credibility of the scalar field, because of the necessary appearance of the dilaton, leading to the realization that the scalar field can, and indeed must, gain a mass and decouple from present-day longrange physics. So, one's point of view on BD cosmology has changed: it does not apply at the present time, but may apply during eras of inflation, or even of quantum gravity, in the early universe. Differences from general relativity may be relevant to the viability of string theory at energies below the Planck scale, and to inflationary scenarios which incorporate scalar fields coupled to the other matter.

However, if one is to use the scalar-tensor theory in the place of general relativity in early cosmology, a natural and necessary first step is the delineation of the full spectrum of cosmological models which are possible in the theory. With the addition of an adjustable coupling constant and a scalar field requiring two initial conditions, it should come as no surprise that a much richer variety of qualitatively distinct models are available here than in general relativity. The purpose of this paper is to consolidate these models into a complete and coherent representation of their character. To our knowledge there has been no previous attempt to do this. An essential practical tool here is a new transformation of variables that reduces the system of equations from fourth order to second order, greatly simplifying the analysis.

After this work was completed, we learned from D. Wands that there is substantial overlap between our work and his Ph.D. thesis [33], including the reduction of the equations to a second order system.

The paper is organized as follows: after writing down the equations for homogeneous and isotropic cosmology in the scalar-tensor theory in Sec. II, we reduce those equations to a 2-dimensional dynamical system in Sec. III. The analysis of the dynamical system, which contains descriptions of all of the qualitatively distinct models allowed by the theory, is performed in Sec. IV. In Sec. V we use the dynamical system formalism to rederive some previously known exact power-law solutions to the theory in flat space, and Sec. VI presents conclusions. 


\section{COSMOLOGY IN BD THEORY}

The field equations for BD theory can be found by varying the action, Eq. (1.1), with respect to the metric and the scalar field. They are

$$
2 \omega \phi^{-1} \square \phi-\omega \frac{\phi^{, \mu} \phi_{, \mu}}{\phi^{2}}+R=0
$$

and

$$
R_{\mu \nu}-\frac{1}{2} g_{\mu \nu} R=8 \pi \phi^{-1} T_{\mu \nu}+\left(\frac{\omega}{\phi^{2}}\right)\left(\phi_{, \mu} \phi_{, \nu}-\frac{1}{2} g_{\mu \nu} \phi^{, \rho} \phi_{, \rho}\right)+\phi^{-1}\left(\phi_{, \mu ; \nu}-g_{\mu \nu} \square \phi\right),
$$

where

$$
\square \phi \equiv \frac{1}{\sqrt{-g}} \frac{\partial}{\partial x_{\mu}}\left(\sqrt{-g} \frac{\partial \phi}{\partial x^{\mu}}\right) .
$$

Taking the trace of Eq. (2.2) and combining the result with Eq. (2.1), we find

$$
\square \phi=\left(\frac{8 \pi}{3+2 \omega}\right) T_{\mu}^{\mu} .
$$

Eqs. (2.2, 2.4) may be viewed as the fundamental equations for BD theory. We take as the background metric the homogeneous and isotropic Friedmann-Robertson-Walker (FRW) line element, which may be written in comoving coordinates as

$$
d s^{2}=-d t^{2}+a^{2}(t)\left[\frac{d r^{2}}{1-k r^{2}}+r^{2} d \Omega_{2}^{2}\right]
$$

where $a(t)$ is the scale factor and $k=0, \pm 1$ is chosen to represent the universe as a space of constant zero, positive or negative curvature, respectively. Then we find that the nontrivial components of the field equations, Eqs. (2.2, 2.4), are

$$
\begin{aligned}
\left(\frac{\dot{a}}{a}+\frac{\dot{\phi}}{2 \phi}\right)^{2}+\frac{k}{a^{2}} & =\left(\frac{2 \omega+3}{12}\right)\left(\frac{\dot{\phi}}{\phi}\right)^{2}+\frac{8 \pi \rho}{3 \phi} \\
-\frac{1}{a^{3}} \frac{d}{d t}\left(\dot{\phi} a^{3}\right) & =\left(\frac{8 \pi}{3+2 \omega}\right) T^{\mu}{ }_{\mu},
\end{aligned}
$$

where we have written only the time component of the metric field equation, since the spatial components are dependent on it through the Bianchi identities. Assuming a perfect fluid form for the stress-energy tensor, i.e., $T_{\mu \nu}=\operatorname{diag}(\rho, p, p, p)$, we also find the usual conservation equation (the time component of $\left.T_{; \nu}^{\mu \nu}=0\right)$ :

$$
\dot{\rho}=-3 \frac{\dot{a}}{a}(p+\rho) \text {. }
$$

Eqs. (2.6-2.8) are the equations for FRW cosmology in BD theory. Note that we have a system of one second-order and two first-order differential equations, so that we need four pieces of initial data to completely specify a solution in this theory, as well as an equation 
of state $p(\rho)$ and values of $\omega$ and $k$. By contrast, when solving for the Friedmann models in general relativity, we have two first-order equations and hence need only two pieces of initial data. The difference stems from the fact that in BD theory, $\phi^{-1}$ acts like a time-varying gravitational constant obeying the second-order Eq. (2.7), so that we need two additional initial conditions in order to close the system. An interesting consequence of this is that even after specifying initial conditions for $a, \phi$ and $\rho$, we are left with a one-parameter family of solutions parametrized by the initial value of the rate of change of the scalar field. Indeed, as has been noted by other authors [15,20], the character of the solutions at early times depends solely upon the value of the constant $a^{3} \dot{\phi}$ at the initial singularity. At later times, however, most solutions become indistinguishable from those where $a^{3} \dot{\phi}=0$ at the singularity. Accordingly, there has been a concentration in the literature on the threeparameter family of solutions characterized by $a^{3}(0) \dot{\phi}(0)=0$. However, one must bear in mind that there has been a loss of generality when this boundary condition is imposed.

We turn now to theoretical considerations regarding the possible values of the coupling constant $\omega$. First, inspection of Eq. (2.6) reveals that at early times, the curvature term vanishes relative to the other terms provided only that $\ddot{a}<0$ as $a \rightarrow 0$, i.e., provided that the initial epoch in the history of the universe was not inflationary. If we make the further, seemingly reasonable assumptions that $\rho>0$ and $\phi>0$, then we see that we must have $\omega \geq-3 / 2$ in order to satisfy Eq. (2.6) for all times. Perhaps a more compelling argument for this constraint on $\omega$ arises when BD theory is reformulated in a way that preserves the form of the Einstein equations (the so-called "Einstein frame"), by treating the scalar as a dynamical field which couples to all of the other matter [21]. When this is done, one finds that the stress-energy for the scalar field is

$$
T_{\mu \nu}^{(\phi)}=\frac{2 \omega+3}{16 \pi G_{0} \phi^{2}}\left(\phi_{, \mu} \phi_{, \nu}-\frac{1}{2} g_{\mu \nu} \phi_{, \rho} \phi^{, \rho}\right),
$$

so that assuming homogeneity,

$$
T_{00}^{(\phi)}=T_{i i}^{(\phi)}=\frac{2 \omega+3}{32 \pi G_{0}}\left(\frac{\dot{\phi}}{\phi}\right)^{2} .
$$

From this, we see that the energy density of the scalar will be nonnegative in the Einstein frame only for $\omega \geq-3 / 2$; thus, for example, one can only prove the existence of black holes or of a cosmological singularity for $\omega$ in this range. As a result of these considerations, we take $\omega \geq-3 / 2$ in what follows.

\section{REDUCTION OF THE FIELD EQUATIONS TO A DYNAMICAL SYSTEM}

In this section, we change variables in order to transform the fourth-order system specified by Eqs. (2.6-2.8) into a pair of coupled second-order equations in which, however, only first derivatives of the new variables appear. The resulting equations may then be analyzed

using the established tools of dynamical systems theory. First we switch to conformal time $d \tau=d t / a(t)$, and define the new variables

$$
\beta \equiv\left(\frac{a^{\prime}}{a}+\frac{\phi^{\prime}}{2 \phi}\right)
$$




$$
\sigma \equiv\left(\frac{2 \omega+3}{12}\right) \frac{\phi^{\prime}}{\phi} \equiv A \frac{\phi^{\prime}}{\phi}
$$

where primes represent derivatives with respect to conformal time, and

$$
A \equiv\left(\frac{2 \omega+3}{12}\right)
$$

Next, we parametrize the equation of state by writing

$$
p=(\gamma-1) \rho
$$

Since the three-form field is necessarily present in the low energy limit of string theory, it is of interest to consider how it might bear on the present study. If we assume a homogeneous and isotropic universe, then it follows that

$$
\begin{aligned}
H_{0 \mu \nu} & =0, \\
H_{123} & =h(t) .
\end{aligned}
$$

If we then use the fact that

$$
T_{\alpha \beta}=\frac{-2}{\sqrt{-g}} \frac{\delta(\sqrt{-g} \mathcal{L})}{\delta g^{\alpha \beta}}
$$

to calculate the stress-energy for the three-form under these assumptions, we find that it can be modelled phenomenologically as a perfect fluid with equation of state $p=\rho$. Then we have, for example,

$$
\gamma= \begin{cases}0, & \text { false-vacuum energy; } \\ 1, & \text { pressureless dust; } \\ 4 / 3, & \text { radiation; } \\ 2, & 3 \text {-form field }\end{cases}
$$

Now we can rewrite Eqs. 2.6 2.8) as

$$
\begin{aligned}
\beta^{2}+k & =\frac{8 \pi \rho a^{2}}{3 \phi}+\frac{\sigma^{2}}{A} \\
\frac{1}{a^{2}}\left(a^{2} \phi^{\prime}\right)^{\prime} & =(1-3 \gamma / 4) \frac{8 \pi \rho a^{2}}{3 A} \\
\rho^{\prime}=-3 \gamma \frac{a^{\prime}}{a} \rho & =-3 \gamma \rho\left(\beta-\frac{\sigma}{2 A}\right)
\end{aligned}
$$

Note that although false-vacuum energy may play a role in the dynamics of the theory, the cosmological constant $\Lambda$ does not explicitly appear in the field equations as we have written them. Unlike in general relativity, where the inclusion of $\Lambda$ is exactly equivalent to the presence of vacuum energy, in BD theory the two are qualitatively distinct. This is the case because whereas in GR the vacuum energy enters the field equations through the term $8 \pi G \rho_{v}$ (which one can equate to $\Lambda$ ), in BD theory it enters through the term $8 \pi \rho_{v} / \phi$, clearly not in general a constant. Others have considered the case $\Lambda \neq 0$ with no matter present 
[22]; in a separate paper, the present authors will consider the system with both $\Lambda \neq 0$ and additional matter present [23].

Combining Eq. (3.8) with Eq. (3.9) and using the definitions of $\beta$ and $\sigma$, we can derive the expression

$$
\sigma^{\prime}=(1-3 \gamma / 4)\left(\beta^{2}+k-\sigma^{2} / A\right)-2 \beta \sigma .
$$

Next we take the conformal time derivative of Eq. (3.8), and use this in conjunction with Eq. (3.10) and Eq. (3.11) to obtain

$$
\beta^{\prime}=(1-3 \gamma / 2)\left(\beta^{2}+k\right)-(3-3 \gamma / 2) \sigma^{2} / A .
$$

Eqs. (3.11, 3.12) constitute a dynamical system (in conformal time) for the variables $\beta$ and $\sigma$, and as such it is conducive to both quantitative and qualitative analysis.

\section{QUALITATIVE ANALYSIS}

Although it is certainly agreeable to have some exact solutions to the system specified by Eqs. (2.6-2.8), such solutions typically correspond to a particular choice of the initial data. Furthermore, it is difficult to synthesize the conglomeration of known particular solutions to the theory into a picture which reveals its overall character. It is therefore desirable to obtain

a more complete understanding of the solution space, which may be accomplished through a qualitative analysis of the dynamical system (3.11, 3.12) in the $\beta-\sigma$ plane, accompanied by a "translation" of the results back into the physically meaningful $a-\phi$ plane. Before beginning the analysis, however, we shall make some general comments regarding the system.

First, let us consider what an equilibrium point of the system represents. Whenever $\beta^{\prime}=\sigma^{\prime}=0$, this implies that $\dot{a}=\beta-\sigma / 2 A$ is a constant, so that

$$
a(t)=\left(\beta-\frac{\sigma}{2 A}\right) t+a_{0} .
$$

Also it is straightforward to show that at such a point,

$$
\phi(t)=C A\left[(\beta-\sigma / 2 A) t+a_{0}\right]^{\sigma /[A(\beta-\sigma / 2 A)]}
$$

unless $\beta=\sigma / 2 A$, in which case

$$
\phi(t)=\phi_{0} \exp \left(\sigma t / A a_{0}\right) .
$$

Hence we see that a fixed point in the $\beta-\sigma$ system represents either a solution where the scale factor changes linearly with time while the scalar varies as a power of time, or a static universe with exponentially varying scalar.

Next, it will be of interest, as we consider the various fixed points of the system, to determine whether or not a given point lies in the physical domain $\rho>0$. We can check this using Eq. (3.8), which reads

$$
\beta^{2}+k=\sigma^{2} / A+\frac{8 \pi \rho a^{2}}{3 \phi},
$$


so that (assuming $\phi>0$,$) any point \left(\beta_{0}, \sigma_{0}\right)$ satisfying

$$
\beta_{0}^{2}+k>\sigma_{0}^{2} / A
$$

will lie in $\rho>0$, whereas points satisfying

$$
\beta_{0}^{2}+k<\sigma_{0}^{2} / A
$$

lie in $\rho<0$ and thus do not represent physical solutions.

A brief discussion regarding equations of state is in order at this point. Of particular interest is the question of what equations of state will lead to inflation in this theory. As was mentioned in Section II, BD theory can be reformulated through a conformal transformation so as to preserve the form of Einstein's equations, while viewing the scalar field simply as a part of the matter (albeit a peculiar part) which couples dynamically to every other field, and hence to the particle masses. Using this form of the theory, we can write the usual equations of general relativity for homogeneous, isotropic cosmology, i.e.,

$$
\begin{aligned}
& \left(\frac{\dot{a}}{a}\right)^{2}+\frac{k}{a^{2}}=\frac{8 \pi G \rho_{t o t}}{3}, \\
& \frac{\ddot{a}}{a}=\frac{-4 \pi G}{3}\left(\rho_{t o t}+3 p_{t o t}\right),
\end{aligned}
$$

where the subscripts are a reminder that these quantities include contributions from the scalar field in addition to the "regular" matter. As we saw previously [cf. Eq. (2.10)], such contributions are given by

$$
p_{\phi}=\rho_{\phi}=\frac{2 \omega+3}{32 \pi G_{0}}\left(\frac{\dot{\phi}}{\phi}\right)^{2} \text {. }
$$

We see that inflation will occur only when $p_{t o t}<-\rho_{t o t} / 3$. For models which are not dominated by the scalar field, this just reduces to the usual condition for inflation in general relativistic cosmology, $p_{m}<-\rho_{m} / 3(\gamma<2 / 3)$. It is also clear, however, that the contribution of the scalar field to the stress-energy tends to act against inflation (and, more generally, against expansion), so that inflation may not occur if the scalar field dynamics become sufficiently dominant, regardless of the equation of state of the other matter.

Finally, the applicability of the singularity theorems of Hawking and Penrose [24] to $\mathrm{BD}$ theory is of interest. In general relativity, these theorems prove the existence of a cosmological singularity for the Friedmann-Robertson-Walker models, provided that Einstein's equations and the strong energy condition are all satisfied. For a perfect fluid, this latter condition reduces to $\rho+3 p \geq 0$, i.e., only for inflationary matter is there the possibility of a cosmological model without a singularity. To apply the theorems to BD theory, we must transform to the Einstein frame, where the Einstein equations are formally preserved. Under this transformation, one finds that 21]

$$
a(t) \rightarrow G_{0}^{-1 / 2} \phi^{-1 / 2} a(t)
$$

from which it follows that 


$$
\left(\frac{\dot{a}}{a}+\frac{\dot{\phi}}{2 \phi}\right)_{\mathrm{BD}} \rightarrow\left(\frac{\dot{a}}{a}\right)_{\mathrm{E}} .
$$

Hence we see that an expanding universe in the Einstein frame is anything with (in our notation) $\beta>0$ in the $\mathrm{BD}$ frame. As a result of this, a model with ordinary matter which appears to contract and then smoothly reexpand in the BD frame will nevertheless be consistent with the singularity theorems so long as $\beta>0$ during the transition, for then the model is in fact always expanding in the Einstein frame and the singularity in the past would be due to the infinite value of the scalar.

Now let us proceed with the analysis. Rewriting the results of section III, we start with

$$
\begin{aligned}
& \beta^{\prime}=\left(1-\frac{3 \gamma}{2}\right)\left(\beta^{2}+k\right)-\left(3-\frac{3 \gamma}{2}\right)\left(\frac{\sigma^{2}}{A}\right), \\
& \sigma^{\prime}=\left(1-\frac{3 \gamma}{4}\right)\left(\beta^{2}+k-\frac{\sigma^{2}}{A}\right)-2 \beta \sigma
\end{aligned}
$$

where, as before,

$$
\begin{aligned}
& \beta \equiv\left(\frac{a^{\prime}}{a}+\frac{\phi^{\prime}}{2 \phi}\right) \\
& \sigma \equiv\left(\frac{2 \omega+3}{12}\right) \frac{\phi^{\prime}}{\phi} \equiv A \frac{\phi^{\prime}}{\phi}
\end{aligned}
$$

and we are in conformal time $d \tau=d t / a$. It is convenient to divide the equilibrium points $\left(\beta_{0}, \sigma_{0}\right)$ into two categories:

$$
\begin{array}{ccc}
\underline{(i)} & \beta_{0}{ }^{2}+k=0, \quad \sigma_{0}=0 & (k=0,-1) \\
\underline{(i i)} & \beta_{0}= \pm \sqrt{\frac{k}{\mu}}(1-3 \gamma / 4), & \sigma_{0}= \pm \sqrt{\frac{k}{\mu}} A(1-3 \gamma / 2)
\end{array}
$$

where

$$
\mu \equiv 3 A(1-\gamma / 2)(1-3 \gamma / 2)-(1-3 \gamma / 4)^{2}
$$

First consider $k=0$, in which case all of the equilibrium points, regardless of the values of $\gamma$ and $\omega$, take on the values $\left(\beta_{0}, \sigma_{0}\right)=(0,0)$. According to our recent discussion, this point represents the static solution $a=a_{0}, \phi=\phi_{0}$. Inspection of the field equations also reveals that this solution is only valid in the true vacuum $\rho=0$. However, although the solution appears at first glance to be unphysical, it may also be viewed as the usual, asymptotically approached solution $\dot{a} \rightarrow 0, a \rightarrow \infty$ for a flat, expanding universe. The fact that the rate of change of the scalar field approaches zero at large times is simply the statement that the strength of the gravitational interaction becomes nearly constant, as it must if the theory is to agree with what we observe in the universe today [25]. Fig. 1a shows the numerically integrated solution curves in the $\beta-\sigma$ plane for a flat, matter-dominated $(\mathrm{p}=0)$ universe, where we have arbitrarily selected $A=1(\omega=9 / 2)$ for the plot. The shaded areas represent unphysical regions of negative energy density, and the dark line 
represents the transition between expansion and contraction, this transition being forbidden for positive energy densities in the present case. The solutions to the right of $\beta=0$ are initially expanding, and we see that they asymptotically approach the equilibrium point $\left(\beta_{0}, \sigma_{0}\right)=(0,0)$, an interpretation of which has already been given. The solutions to the left of $\beta=0$ are initially contracting, and will continue to do so until a singularity is reached. We see that the solutions approach and depart from equilibrium along a particular path, which represents a known power-law solution to the flat-space field equations. We shall consider such exact solutions, and their relation to the general class of cosmological models, in Section V. For the time being, we simply mention that the attractive nature of such solutions near equilibrium is a general feature of the theory, and that these solutions typically correspond to the particular choice of initial data $a^{3} \dot{\phi}=0$ at the singularity.

A qualitative change occurs when the coupling constant takes on a value in the range $-3 / 2 \leq \omega \leq 0$. Fig. $1 \mathrm{~b}$ is similar to Fig. 1a, except that here we take $\omega=-1 / 2$. Note that now the line representing $\dot{a}=0$ exists in the physical regime where $\rho>0$. In fact, one sees that models exist where the universe is initially contracting, but then makes a smooth transition to expansion without meeting a singularity. This behavior has been noted by other authors [19], and models with this feature are sometimes called "bounce" universes. It should be mentioned that such models are present for $\omega=-1$, the value corresponding to the low-energy limit of string theory.

Next we examine the flat space models with an inflationary equation of state. Fig. 1c shows the solution curves for $k=0$ and $p=-\rho$, where again we arbitrarily select $\omega=$ $9 / 2$. The attracting nature of the particular solution represented by the line $\beta=3 \sigma$ is quite apparent, and this is in fact the inflationary power-law solution which has been found previously by other authors [26]. Here all expanding universes asymptotically approach this state, and once again all contracting universes must reach a singularity, although we see that as they do so, they approach the true-vacuum solutions (represented by the dark lines at the border of the shaded region). These are examples of what may be termed ' $\phi$-dominated' models; i.e., models where the scalar field dynamics overwhelm the effect of the other matter and thus attract the universe towards true-vacuum behavior. Nevertheless, these models must contract until a singularity is reached.

Again the character of the solutions changes in the range $-3 / 2 \leq \omega \leq 0$, in a manner similar to the noninflationary case. Here, however, not only do we see that there exist "bounce" models, but we also find that inflation is no longer possible in this range of $\omega$. Rather, all models approach the equilibrium solution $\dot{a}=0, a \rightarrow \infty$ as they would for noninflationary equations of state; another example of ' $\phi$-domination'. This behavior is illustrated in Fig. 1d. In the range $0<\omega \leq 1 / 2$, inflation is still not possible, although the "bounce" models are absent.

Now let us consider the case of the open universe, $k=-1$. First consider the equilibrium points in category $(\mathrm{i})$, i.e., $\left(\beta_{0}, \sigma_{0}\right)=( \pm 1,0)$. Here the corresponding solutions are $a(t)=a_{0} \pm t, \phi(t)=\phi_{0}$ which are, again by inspection of the field equations, valid only for $\rho=0$. The physical interpretation of the point $\left(\beta_{0}, \sigma_{0}\right)=(+1,0)$ is straightforward; we can view it either as representing a particular vacuum solution, or as the asymptotically approached endpoint of an open, expanding universe. The contracting solution represented by the point $\left(\beta_{0}, \sigma_{0}\right)=(-1,0)$ does not lend itself to interpretation quite so easily; to aid us in our efforts we can perform a stability analysis. This proceeds by linearization, i.e., we 
check the eigenvalues of the Jacobian matrix for the system $\beta^{\prime}=f(\beta, \sigma) ; \sigma^{\prime}=g(\beta, \sigma)$. In cases where the eigenvalues of the Jacobian all have nonvanishing real part, the fixed point is called hyperbolic and we can determine its stability from the signs of those real parts: if the real part of each of the eigenvalues is negative at a given equilibrium point, the solution is stable at that point; if the real part of each eigenvalue is positive, or if the real part of one eigenvalue is positive and that of the other is negative, then the solution is unstable at that point. Finally, if the real part of any of the eigenvalues is zero at a point, then the point is called nonhyperbolic and its stability in the neighborhood of that point cannot be determined by this method [27]. Hence we write

$$
\left(\begin{array}{c}
\xi^{\prime} \\
\eta^{\prime}
\end{array}\right)=\underbrace{\left(\begin{array}{cc}
2 \beta_{0}(1-3 \gamma / 2) & -\frac{2 \sigma_{0}}{A}(3-3 \gamma / 2) \\
2 \beta_{0}(1-3 \gamma / 4)-2 \sigma_{0} & -\frac{2 \sigma_{0}}{A}(1-3 \gamma / 4)-2 \beta_{0}
\end{array}\right)}_{\text {Jacobian }}\left(\begin{array}{l}
\xi \\
\eta
\end{array}\right)+\ldots
$$

where $\xi \equiv \beta-\beta_{0}$ and $\eta \equiv \sigma-\sigma_{0}$, and diagonalize the Jacobian case by case. In this manner we find that for the equilibrium points in the $k=-1$ models under consideration,

$$
\begin{aligned}
& \text { if }\left(\beta_{0}, \sigma_{0}\right)=\left\{\begin{array}{c}
(1,0) \\
(-1,0)
\end{array}\right\}, \quad \text { then } \\
& \left\{\begin{array}{l}
\lambda_{1}=2(1-3 \gamma / 2), \lambda_{2}=-2 \\
\lambda_{1}=2(3 \gamma / 2-1), \lambda_{2}=2
\end{array}\right\} \Rightarrow\left\{\begin{array}{l}
\text { stable, } \gamma>2 / 3 \\
\text { unstable, } \gamma \neq 2 / 3
\end{array}\right\} .
\end{aligned}
$$

As expected, the point $(1,0)$ represents a stable solution for noninflationary, open universes. The point $(-1,0)$, although an equilibrium point of the dynamical system, in general represents an unstable and thus unphysical contracting solution. Hence a small perturbation will send the universe along a more rapidly contracting trajectory as shown in Fig. 2a. In any case, all models with $\omega>0$, negative curvature and noninflationary equations of state must, if initially expanding, approach equilibrium at $\dot{a}=0, a \rightarrow \infty$ or, if initially contracting, reach a singularity. If the equation of state is inflationary, i.e., if $\gamma<2 / 3$, then the expanding equilibrium solution represented by $(1,0)$ becomes unstable, and in the range $\omega>1 / 2$ we do in fact see inflation for these models. Although the existence and stability of the equilibrium points $( \pm 1,0)$ for $k=-1$ do not depend upon the value of $\omega$, we again find models which can contract and smoothly reexpand if $-3 / 2 \leq \omega \leq 0$. This behavior is illustrated in Fig. $2 \mathrm{~b}$ for models dominated by pressureless dust. For $k=-1$ models with $-3 / 2 \leq \omega \leq 1 / 2$ and inflationary equations of state, inflation does not occur despite the fact that the equlibrium solution at $(1,0)$ is unstable. This is illustrated in Fig. 2c and Fig. 2d. In order to better understand these models, we must move on to an analysis of the remaining class of equilibrium points.

Let us consider, then, case (ii) of Eq. (4.11), i.e., the equilibrium points with $\sigma_{0} \neq 0$. Referring back to that equation, we find that such points will exist only if the condition $k / \mu(\omega, \gamma)>0$ is met, which implies that

$$
\frac{4}{3}\left[1-\left(\frac{2 \omega+3}{8 \omega}\right)^{1 / 2}\right]<\gamma<\frac{4}{3}\left[1+\left(\frac{2 \omega+3}{8 \omega}\right)^{1 / 2}\right]
$$


if $k=-1$, and

$$
\gamma>\frac{4}{3}\left[1+\left(\frac{2 \omega+3}{8 \omega}\right)^{1 / 2}\right] \quad \text { or } \quad \gamma<\frac{4}{3}\left[1-\left(\frac{2 \omega+3}{8 \omega}\right)^{1 / 2}\right],
$$

if $k=+1$. We can thus think of the curves

$$
\gamma=\frac{4}{3}\left[1 \pm\left(\frac{2 \omega+3}{8 \omega}\right)^{1 / 2}\right]
$$

as bifurcation curves in the parameter space $(\omega, \gamma)$. By this one means simply that there are equilibrium points of the dynamical system which move in or out of existence as those curves are crossed. However, using Eq. (3.8) and Eq. (4.11), we find that these fixed points will lie in the domain $\rho>0$ only if $\gamma<2 / 3$, i.e., only for matter with an inflationary equation of state. Fig. 3 shows the bifurcation curves in the $\omega-\gamma$ plane. The region between the curves contains new equilibrium points for $k=-1$; the regions exterior to the curves contains such points for $k=+1$. The unshaded region represents that part of the parameter space with equations of state which can support the solutions represented by these new fixed points and still maintain $\rho>0$. It is evident that the only bifurcation of physical interest occurs for $\gamma=0$, i.e., with vacuum energy dominant. In that case, one finds that two new equilibrium points appear at $\omega=1 / 2$. If the curvature is positive $(k=+1)$, then these equilibrium points exist in the regime $\omega>1 / 2$; if the curvature is negative $(k=-1)$, then they exist in the regime $\omega<1 / 2$. Let us first complete our analysis of the negative curvature models. Taking $k=-1$ in Eq. (4.11), we have

$$
\left(\beta_{0}, \sigma_{0}\right)=\left( \pm(1-3 A)^{-1 / 2}, \pm A(1-3 A)^{-1 / 2}\right) \quad(\omega<1 / 2)
$$

for which we find from the Jacobian that

$$
\left(\beta_{0}, \sigma_{0}\right)=\left\{\begin{array}{c}
(+,+) \\
(-,-)
\end{array}\right\} \Rightarrow\left\{\begin{array}{ll}
\lambda_{1}<0, & \lambda_{2}<0 \\
\lambda_{1}>0, & \lambda_{2}>0
\end{array}\right\}
$$

Here we see that one of the equilibrium points is stable (a sink) while the other is unstable (a source), as we have already seen explicitly in Fig. 2c and Fig. 2d. Let us consider the nature of the stable solution. Using the definitions of $\beta$ and $\sigma$, one finds that the solution at this point is given by

$$
\begin{aligned}
& a(t)=a_{0}+(\Sigma / 2) t \\
& \phi(t)=C a^{2}
\end{aligned}
$$

where

$$
\Sigma \equiv(1-3 A)^{-1 / 2}
$$

and, by assumption, $\rho=\rho_{v a c}=$ const. Hence, although not inflationary, the universe will expand forever as we would expect for a model with negative curvature. Also, one finds that in order to satisfy the field equations, the value of $\rho_{v a c}$ must be given by 


$$
\rho_{v a c}=\frac{3 A C}{4 \pi(3 A-1)}=\frac{(2 \omega+3) C}{4 \pi(1-2 \omega)},
$$

where $C$ is the integration constant in the the solution for $\phi$, which we can write as $C=$ $\phi_{0} / a_{0}^{2}$. Hence we see that for this solution, the vacuum energy is completely determined by the initial conditions of the scale factor and the scalar field, and by $\omega$.

Finally let us consider the models with positive curvature, i.e., those with $k=+1$. The analysis of the equilibrium points is, in light of our previous discussion, particularly simple. There are no equilibrium points in category (i) of Eq. (4.11), and the equilibrium points from category (ii) which lie in the domain $\rho>0$ exist only for inflationary equations of state and only for $\omega>1 / 2$. Then using Eq. (4.11), we find

$$
\left(\beta_{0}, \sigma_{0}\right)=\left( \pm(3 A-1)^{-1 / 2}, \pm A(3 A-1)^{-1 / 2}\right) \quad(\omega>1 / 2),
$$

and diagonalization of the Jacobian at those points yields

$$
\left(\beta_{0}, \sigma_{0}\right)=\left\{\begin{array}{c}
(+,+) \\
(-,-)
\end{array}\right\} \Rightarrow\left\{\begin{array}{ll}
\lambda_{1}<0, & \lambda_{2}>0 \\
\lambda_{1}>0, & \lambda_{2}<0
\end{array}\right\},
$$

i.e., both of the equilibrium points are unstable. So for models with positive curvature, we have no physically interesting equilibrium points regardless of the equation of state and the value of $\omega$. Nevertheless, we may determine the character of the solutions from their evolution in the $\beta-\sigma$ plane. In general, one expects that such models should recollapse unless inflation occurs. Indeed, one finds that for noninflationary equations of state, i.e., $\gamma>2 / 3$, and in the range $\omega>0$, this is in fact the case. However, as before, models with $-3 / 2 \leq \omega \leq 0$ and those with $\gamma<2 / 3$ have features unique to this theory. To begin with, one finds that in the range $-3 / 2 \leq \omega \leq 0$, there exist noninflationary models which do not collapse, despite having positive curvature. This is illustrated for pressureless dust in Fig. 4a. Note that the initial conditions must be carefully tuned for such models, and in particular that $\sigma<0$ always, so that $\phi$ is a monotonically decreasing function of time.

For inflationary matter and positive curvature, consider first the range $-3 / 2 \leq \omega \leq 0$. Here one finds that inflation never occurs, although there exist models with carefully chosen initial conditions which expand forever. All other models, either initially expanding or initially contracting, collapse to a singularity. These models are illustrated in Fig. 4b. Next, we consider vacuum energy-dominated models with $k=+1$ but with $0<\omega<$ $1 / 2$. Here we find that inflation never occurs, and furthermore that all models, regardless of initial conditions, collapse to a singularity. Finally, consider inflationary models with positive curvature and in the range $\omega>1 / 2$, where we have seen that there exist 2 unstable equilibrium points in the $\beta-\sigma$ plane. Here we find solutions with several interesting features. First, in the range $1 / 2<\omega<9 / 2$, there are models which start out expanding, and then recollapse rather than inflate, including some which "coast" at $\dot{a}=0$ before beginning their contraction. If $\omega>9 / 2$, then in addition we find that there exist "bounce" models which start out contracting, but then smoothly reexpand and inflate - these are similiar to general relativistic de Sitter spacetime in $k=+1$ coordinates. Also in this range of $\omega$, there are models which are initially contracting, then smoothly reexpand, and finally recontract until a singularity is reached — we call these "vacillating universes." Indeed, there exist models which are initially expanding, then recontract, and at last smoothly reexpand and inflate. 
If $\omega \geq 9 / 2$, then in general one finds that the universe will inflate if and only if its initial conditions lie in the region bounded by the stable manifolds of the two equilibrium points. On the other hand, any model which has initial conditions which lie between either of the stable manifolds and the true vacuum in the $\beta-\sigma$ plane will collapse back to a singularity. The fact that the stable manifolds extend arbitrarily far back in the negative $\beta$-direction gives rise to yet another feature unique to this theory: an arbitrarily rapidly contracting universe will slow down, smoothly reexpand and inflate if the energy of the scalar field is chosen just right. These models represent a particularly glaring form of " $\phi$-domination": whether the universe collapses to a singularity or inflates depends solely upon the energy of the scalar relative to the expansion rate. Fig. 4c illustrates this with $\omega=10.5$. The division of the $\beta-\sigma$ plane into inflationary and noninflationary cells by the stable manifolds is apparent. Fig. $4 \mathrm{~d}$ is a closeup of the neighborhood of the equilibrium point in the expanding regime of Fig. 4c, and one sees explicitly the "bounce" and the "vacillating" universe models. The limiting cases of "vascillation" are clearly present as well. For example, a universe which slows down in its expansion (or contraction), and then speeds back up may be termed a "hesitation" model, whereas one which just stops but then continues its expansion after a finite time may be called a "coasting" model. The potentially drastic effects of the scalar field can also be seen in the special case of $\omega=9 / 2$, where one finds that a necessary condition for inflation to occur is $\beta>\sigma$, which implies that

$$
\left(\frac{\dot{a}}{a}\right)_{0}>\frac{1}{2}\left(\frac{\dot{\phi}}{\phi}\right)_{0} .
$$

(However, even if this relation is satisfied, the universe may recollapse if $\dot{\phi} / \phi$ is negative enough initially.) For this value of $\omega$, a universe which is initially static will begin to expand, following one of the stable manifolds towards its unstable equilibrium point, although a small perturbation in the metric or the scalar will cause either collapse to a singularity or inflation, depending upon the direction of the perturbation.

\section{EXACT SOLUTIONS FOR FLAT SPACE}

Many exact solutions to Eqs. (2.6-2.8) have been found for the case of flat space $(k=0)$, some of which yield power-laws for the evolution of the scale factor, the scalar field, and the energy density [28]. Here we find exact power-law solutions which are parametrized by the equation of state, and show that these include all possible power-law solutions to the field equations.

If we define

$$
\lambda \equiv \frac{\sigma}{\beta},
$$

then using Eqs. (3.11, 3.12) with $k=0$, we can derive the expression

$$
\frac{d \lambda}{d \ln \beta}=\frac{\left(\lambda^{2}-A\right)[3 \lambda(1-\gamma / 2)-(1-3 \gamma / 4)]}{A(1-3 \gamma / 2)-3 \lambda^{2}(1-\gamma / 2)} .
$$

Note that any constant value of $\lambda$ which is a root of the cubic polynomial in the numerator of the right-hand-side will satisfy this equation. Those roots are 


$$
\begin{aligned}
& \lambda= \pm \sqrt{A} \\
& \lambda=\frac{(1-3 \gamma / 4)}{3(1-\gamma / 2)}
\end{aligned}
$$

the first of which represent the true vacuum solutions, and the last of which represents a solution with matter (or vacuum energy) present. Before finding these solutions explicitly, let us remark that the initial condition $\dot{\phi} a^{3} \rightarrow 0$ as $a \rightarrow 0$ is implicit in most of the solutions that we can generate with this formalism. To see this, first note that if follows from the definitions of $\beta$ and $\sigma$ that if $\lambda$ is a constant, then

$$
\phi=\phi_{0}\left(\frac{a}{a_{0}}\right)^{\alpha}
$$

where we have set $\phi=\phi_{0}$ when $a=a_{0}$, and

$$
\alpha \equiv \frac{\lambda}{A-\lambda / 2}
$$

Then we can write

$$
\dot{\phi} a^{3} \sim \dot{a} a^{\alpha+2}
$$

from which we see that if $a \sim t^{C_{1}}$ at early times, then $\dot{\phi} a^{3} \rightarrow 0$ as $a \rightarrow 0$ so long as

$$
C_{1}>\frac{1}{3+\alpha}
$$

One can easily verify that this relation is satisfied for all physically reasonable perfect fluid equations of state, i.e., for $\gamma=0,1$, or $4 / 3$, unless:

$$
\begin{aligned}
& -5 / 6<\omega<-1 / 2 \text { for } \gamma=0 \\
& -4 / 3<\omega<-1 \text { for } \gamma=1 .
\end{aligned}
$$

To see that the $\lambda=$ constant solutions give us all possible power-law solutions, assume a power-law solution $\phi \propto t^{p}, a \propto t^{q}$. It then follows from the definitions of $\beta$ and $\sigma$ that

$$
\beta=\left(\frac{q+p / 2}{p}\right) \frac{\sigma}{A}
$$

i.e., $\lambda=$ constant.

\section{A. True-Vacuum Solutions}

If we set $\lambda= \pm \sqrt{A}$, we obtain the true-vacuum solutions. This can be seen by using Eq. (5.5) and setting $\rho=0$ and $k=0$ in Eqs. (2.6, 2.7), upon which we find that for $\lambda= \pm \sqrt{A}$, Eq. (2.6) is satisfied identically, whereas Eq. (2.7) can be integrated to yield the power-law solutions 


$$
\begin{aligned}
& a(t)=a_{0}\left[1+\left(t / t_{0}\right)\right]^{( \pm 2 \sqrt{A}-1) /( \pm 6 \sqrt{A}-1)}, \\
& \phi(t)=\phi_{0}\left[1+\left(t / t_{0}\right)\right]^{2 /( \pm 6 \sqrt{A}-1)}
\end{aligned}
$$

where we have set $\phi(t=0)=\phi_{0}$ and $a(t=0)=a_{0}$, and $t_{0}$ is arbitrary. These solutions are not valid for $\omega=-4 / 3$, since the exponents corresponding to the positive roots in Eq. (5.11) blow up there. The special true-vacuum solution corresponding to $\omega=-4 / 3$ was found by O'Hanlon and Tupper in [18] to be deSitter in nature, and may be written in the form

$$
\begin{aligned}
& a(t)=a_{0} \exp \left(t / t_{0}\right), \\
& \phi(t)=\phi_{0} \exp \left(-3 t / t_{0}\right) .
\end{aligned}
$$

\section{B. Solutions with Matter Present}

Now consider the solutions where $\rho \neq 0$. Then from $T_{; \nu}^{0 \nu}=0$ we have

$$
\begin{aligned}
\frac{\dot{\rho}}{\rho} & =-3 \gamma \frac{\dot{a}}{a} \\
\rho & =\rho_{0}\left(\frac{a}{a_{0}}\right)^{-3 \gamma},
\end{aligned}
$$

where we have set $\rho=\rho_{0}$ when $a=a_{0}$. Using Eqs (5.5, 5.13) in Eqs (2.6, 2.7), we find that they become

$$
\left[(1+\alpha / 2)^{2}-A \alpha^{2}\right]\left(\frac{\dot{a}}{a}\right)^{2}=\frac{8 \pi \rho_{0}\left(a / a_{0}\right)^{-3 \gamma-\alpha}}{3 \phi_{0}}
$$

and

$$
\ddot{\phi}+3 \dot{\phi} \frac{\dot{a}}{a}=\frac{8 \pi(4-3 \gamma) \rho_{0}\left(a / a_{0}\right)^{-3 \gamma}}{3+2 \omega} .
$$

Again setting $a(t=0)=a_{0}$ and $\phi(t=0)=\phi_{0}$, Eq. (5.14) immediately integrates to

$$
a(t)=a_{0}[1+\chi t]^{2 /(\alpha+3 \gamma)}
$$

where

$$
\chi \equiv\left(\frac{\alpha+3 \gamma}{2}\right)\left(\frac{8 \pi \rho_{0}}{3 \phi_{0}\left[(1+\alpha / 2)^{2}-A \alpha^{2}\right]}\right)^{1 / 2} .
$$

Now using Eq. (5.5) and Eq. (5.16), we see that

$$
\phi(t)=\phi_{0}(1+\chi t)^{2 \alpha /(\alpha+3 \gamma)} .
$$

Also, using Eq. (5.13), we find

$$
\rho(t)=\rho_{0}(1+\chi t)^{-6 \gamma /(\alpha+3 \gamma)}
$$


Eqs. (5.16 5.19) specify all power-law solutions to flat space FRW/BD cosmology.

Now we can use these results for the various types of matter. First consider vacuum energy, where $p=-\rho_{v a c}$ is a constant, $\gamma=0, \lambda=1 / 3$, and $\alpha=4 /(2 \omega+1)$. Then using Eqs. (5.165.19), we find

$$
\begin{aligned}
& a(t)=a_{0}(1+\chi t)^{\omega+1 / 2} \\
& \phi(t)=\phi_{0}(1+\chi t)^{2}
\end{aligned}
$$

with

$$
\chi_{v a c}^{2}=\frac{32 \pi \rho_{v a c}}{\phi_{0}(6 \omega+5)(2 \omega+3)}
$$

in agreement with the known power-law solutions [6,26]. Note that if $\omega \leq 1 / 2$, then $\ddot{a} \leq 0$ and inflation does not occur. This is noteworthy, as we see that the bare low-energy limit of string theory, corresponding as it does to a value of $\omega=-1$, does not permit an inflationary solution for flat space. In Section IV, we saw that this result also holds for a universe with curvature.

Next consider a MD era, where $p=0, \gamma=1, \lambda=1 / 6$, and $\alpha=1 /(\omega+1)$. We find

$$
\begin{aligned}
& a(t)=a_{0}(1+\chi t)^{(2 \omega+2) /(3 \omega+4)} \\
& \phi(t)=\phi_{0}(1+\chi t)^{2 /(3 \omega+4)} \\
& \rho(t)=\rho_{0}(1+\chi t)^{-3(2 \omega+2) /(3 \omega+4)}
\end{aligned}
$$

where

$$
\chi_{\mathrm{MD}}^{2}=\frac{4 \pi \rho_{0}}{\phi_{0}}\left(\frac{3 \omega+4}{2 \omega+3}\right)
$$

again in agreement with the known power-law solutions $[7,20 \rrbracket$.

Finally, for a RD era, we have $p=\rho / 3, \gamma=4 / 3, \lambda=0$, and $\alpha=0$. From this we find

$$
\begin{aligned}
& a(t)=a_{0}(1+\chi t)^{1 / 2} \\
& \phi(t)=\phi_{0}(1+\chi t)^{-1 / 2}, \\
& \rho(t)=\rho_{0}(1+\chi t)^{-2}
\end{aligned}
$$

where

$$
\chi_{\mathrm{RD}}^{2}=\frac{32 \pi \rho_{0}}{3 \phi_{0}}
$$

Note that in this case, the scale factor and energy density evolve just as they do in ordinary general relativity, independent of the coupling constant $\omega$. This is the case because the scalar field has been effectively decoupled from the other matter due to the vanishing trace of the stress-energy tensor [cf. Eq. (2.4)]. 


\section{CONCLUSIONS}

The resurgence of interest in scalar-tensor gravity is justified, in part, by the formal similarity of the theory to low-energy string theory, and by the power-law inflationary solution which ameliorates the graceful exit problem of some general relativistic models of inflation. However, in order to create a viable cosmological model with the scalar-tensor theory, one must understand, to as full an extent as possible, what models are available in the theory and how those with qualitative differences may be distinguished from one another. Here we have shown that there exist a plethora of models which differ substantively from those present in the standard general relativistic cosmology. In general, the models are parametrized by initial conditions of the scalar field, the value of the BD coupling constant, an initial value of the Hubble parameter, and an initial density and equation of state of the matter present. The first two parameters are not, of course, present in general relativity.

For flat space, in addition to the standard models which start with a singularity at the big bang and then asymptotically approach $\dot{a}=0$ (or the time-reversal of this behavior) as in general relativity, we have found "bounce" models, which pass smoothly from contraction to expansion. Such models are present for all types of matter in the range of coupling constant $\omega<0$. In addition, we have found that a flat-space inflationary solution does not exist in this theory in the range $\omega \leq 1 / 2$, irrespective of initial conditions.

For a universe with negative curvature, we again find a set of models which have an analogue in general relativity, in this case those which start with a singularity and then asymptotically approach the solution $a \sim t$ (again the time-reversal of this exists with $t \rightarrow-t$ ). And again, we find "bounce" models when $\omega<0$, as well as the absence of an inflationary solution for $\omega<1 / 2$. We find that in this latter range of $\omega$, all expanding negative curvature models with inflationary-type matter present approach a particular solution at late times, where $a(t) \sim t$ and $\phi(t) \sim t^{2}$.

For a universe with positive curvature, the standard models are once more present, but anomalous behavior occurs both in the range $-3 / 2 \leq \omega \leq 0$ and for inflationary matter. Again inflation will not occur if $\omega<1 / 2$, in which case all models with vacuum energy dominant collapse to a singularity only in the range $0<\omega<1 / 2$. If $\omega$ is negative, there exist models which perpetually expand for all types of matter, including vacuum energy. If vacuum energy is present and $\omega>1 / 2$, we find several classes of models which have no analogues in general relativity without the addition of a cosmological constant [29]. First, inflation still does not occur for certain ranges of initial conditions, those ranges being determined by the locations of the stable manifolds of the dynamical system in our formalism, which in turn are determined by the value of $\omega$. Next we find "hesitating" and "coasting" models, which start with a big bang and either slow down and "hesitate" before continuing their expansion, or "coast" at a quasistatic value of $a(t)$. When $\omega>9 / 2$, we find models which expand from a big bang, contract for a time and then reexpand ("vacillating universes" - the time-reversed solution also exists) and models which pass from "deflation"

to inflation, i.e., universes which, although contracting arbitrarily fast, turn around and ultimately inflate.

Of particular interest are those models with nonzero curvature that are dominated by vacuum energy which, contrary to the well-accepted inflationary dogma, do not approach a corresponding flat-space solution at late times. Indeed, we have seen that there exist, for all 
values of the coupling constant, vacuum energy-dominated models with curvature for which there are no corresponding flat-space solutions at all. In such cases, the initial energy of the scalar field relative to the expansion rate sometimes plays a key role in determining whether inflation will occur, a fact which appears to have been largely overlooked in the literature.

\section{ACKNOWLEDGEMENTS}

This research was supported in part by the National Science Foundation under Grants

No. PHY89-04035 and PHY90-08502. We are grateful to J. Barrow and D. Wands for helpful communications. 


\section{REFERENCES}

[1] A.H. Guth, Phys. Rev. D 23 (1981), 347.

[2] E.W. Kolb and M.S. Turner, The Early Universe (Addison-Wesley Pub. Co., New York, 1990), Chapter 8.

[3] A.H. Guth and E.J. Weinberg, Nucl. Phys. B212 (1983), 321; W. Hawking, I. Moss, and J. Stewart, Phys. Rev. D 26 (1982), 2681.

[4] A.D. Linde, Phys. Lett. 108B (1982), 389; A. Albrecht and P.J. Steinhardt, Phys. Rev. Lett. 48 (1982), 1220.

[5] A.D. Linde, Particle physics and inflationary cosmology (Harwood Academic Publishers, New York, 1990).

[6] D.A. La and P.J. Steinhardt, Phys. Rev. Lett. 62 (1989), 376.

[7] C. Brans and R.H. Dicke, Phys. Rev. 124 (1961), 925.

[8] E.J. Weinberg, Phys. Rev. D 40 (1989), 3950.

[9] R.D. Reasenberg et al, Astrophys. Jour. Lett. (1979), 234, L219.

[10] D. La, P.J. Steinhardt and E. Bertschinger, Phys. Lett. 231B (1989), 231.

[11] A.R. Liddle and D.H. Lyth, Phys. Lett. B 291 (1992), 391.

[12] P.J. Steinhardt and F.S. Accetta, Phys. Rev. Lett. 64 (1990), 2740.

[13] R. Holman, E.W. Kolb, S. Vadas and Y. Wang, Phys. Rev. D 43 (1991), 3833.

[14] See, for example, C.G. Callan, D. Friedan, E.J. Martinec and M.J. Perry, Nucl. Phys. $B 262$ (1985), 597.

[15] G.S. Greenstein, Ap. Space Sci. 2 (1968), 155.

[16] H. Nariai, Progr. Theor. Phys. 42 (1968), 544.

[17] R.E. Morganstern, Phys. Rev. D 4 (1971), 278.

[18] J. O'Hanlon and B.O.J. Tupper, Nuovo Cimento 7B (1972), 305.

[19] L.E. Gurevich, A.M. Finkelstein and V.A. Ruban, Ap. Space Sci. 22 (1973), 231.

[20] S. Weinberg, in Gravitation and Cosmology: Principles and Applications of the General Theory of Relativity (John Wiley and Sons, New York, 1972), Section 16.4.

[21] R.H. Dicke, Phys. Rev. 125 (1962), 2163.

[22] C. Romero and A. Barros, Gen. Rel. and Grav. 23 (1993), 491.

[23] S. Kolitch and D. Eardley, in preparation.

[24] S.W. Hawking and R. Penrose, Proc. R. Soc. London A314 (1969), 529.

[25] For a complete list of references regarding experimental tests of the constancy of the gravitational "constant", see C.M. Will, Intl. Jour. Mod. Phys. D 1 (1992), 50.

[26] This solution was first found by C. Mathiazhagan and V.B. Johri, in Class. Quantum Grav. 1 (1984), L29, although they did not realize at the time that it offered a solution to the exit problem.

[27] For a pedagogical review of the stability analysis of dynamical systems, see, for example, M.W. Hirsch and S. Smale, Differential Equations, Dynamical Systems and Linear Algebra (Academic Press, New York, 1974).

[28] An extensive catalogue of exact solutions to the BD equations for cosmology can be found in the paper by D. Lorenz-Petzold, in Solutions of Einstein's Equations: Techniques and Results, eds. C. Hoenselaers and W. Dietz (Springer-Verlag, Berlin, 1984).

[29] If one adds a cosmological constant to general relativity, however, much of this behavior is manifested in the resulting models. See, for example, J.E. Felten and R. Isaacman, Rev. Mod. Phys. 58 (1986), 689. 
[30] J.D. Barrow, Phys. Rev. D 47 (1993), 5329; Phys. Rev. D 48 (1993), 3592.

[31] J.D. Barrow and K. Maeda, Nucl. Phys. B 341 (1990), 294.

[32] T. Damour and K. Nordvedt, Phys. Rev. Lett. 70 (1993), 2217; Phys. Rev. D 48 (1993), 3436.

[33] Wands, D. Unpublished Ph.D. Thesis, University of Sussex, 1993. 


\section{FIGURES}

FIG. 1. The evolution of solutions in the $\beta-\sigma$ plane for flat space. (a) Matter is $\gamma=1$ (pressureless dust); and $\omega=9 / 2$, representative of $\omega>0$. Solutions to the right of the line $\beta=\sigma / 2$ represent expanding universes; those to the left are contracting universes. The shaded regions require negative energy density and so are disallowed physically. (b) Same as (a), except $\omega=-1 / 2$. The line $\frac{d a}{d t}=0$ has moved into the regime of positive energy density, so that some models pass smoothly from contraction to expansion, a general feature of models with $-3 / 2 \leq \omega \leq 0$. (c) Flat space $\gamma=0$ (vacuum energy dominated) models, with $\omega=9 / 2$. All initially expanding universes become inflationary - they flow out along the prominent channel to the left — regardless of initial conditions when $k=0$ and $\omega>1 / 2$. (d) Flat space, $\gamma=0$ (vacuum energy dominated) models with $\omega=-1 / 2$, representative of $-3 / 2 \leq \omega \leq 0$. Inflation does not occur, although all initially expanding universes continue to expand forever.

FIG. 2. Some models with $k=-1$ (negative curvature). (a) $\gamma=1$ (pressureless dust) with $\omega>0$. (b) $\gamma=1$ with $\omega<0$. (c) $\gamma=0$ (vacuum energy) with $\omega<0$. (d) a closeup of the stable equilibrium point in (c). In (a) $\omega=9 / 2$; in (b-d) $\omega=-1 / 2$.

FIG. 3. Bifurcation diagram in the parameter space $\omega-\gamma$. The shaded region is disallowed physically as it requires $\rho<0$. The regions exterior to the curves contain bifurcated equilibrium points for $k=+1$; the region interior to the curves contains bifurcated equilibrium points for $k=-1$. The bifurcation for $k=+1$ and $\gamma=0$ (vacuum energy) is physically permissible and occurs when $\omega=1 / 2$.

FIG. 4. Some models with $k=+1$ (positive curvature). (a) $\gamma=1$ (pressureless dust) and $\omega=-1 / 2$, representative of $-3 / 2<\omega<0$. Some initially expanding models do not recollapse, despite positive curvature. (b) $\gamma=0$ (vacuum energy dominant); and $\omega=-1 / 2$, representative of $-3 / 2<\omega<0$. Most expanding solutions recollapse; a few expand forever, but inflation never occurs. (c) $\gamma=0$ (vacuum energy dominant); and $\omega=10.5$, representative of $\omega>9 / 2$. Most expanding universes inflate, but some do not. Here an arbitrarily rapidly contracting universe will slow down, stop and inflate if the energy of the scalar relative to the expansion rate is carefully chosen. (d) A closeup of an unstable equilibrium point in (c). The stable manifold separates models which inflate from those which recollapse. Also apparent are "vacillation" universes which start out contracting, then briefly expand, and finally recontract to a singularity. 
This figure "fig1-1.png" is available in "png" format from: http://arxiv.org/ps/gr-qc/9405016v2 
This figure "fig1-2.png" is available in "png" format from: http://arxiv.org/ps/gr-qc/9405016v2 

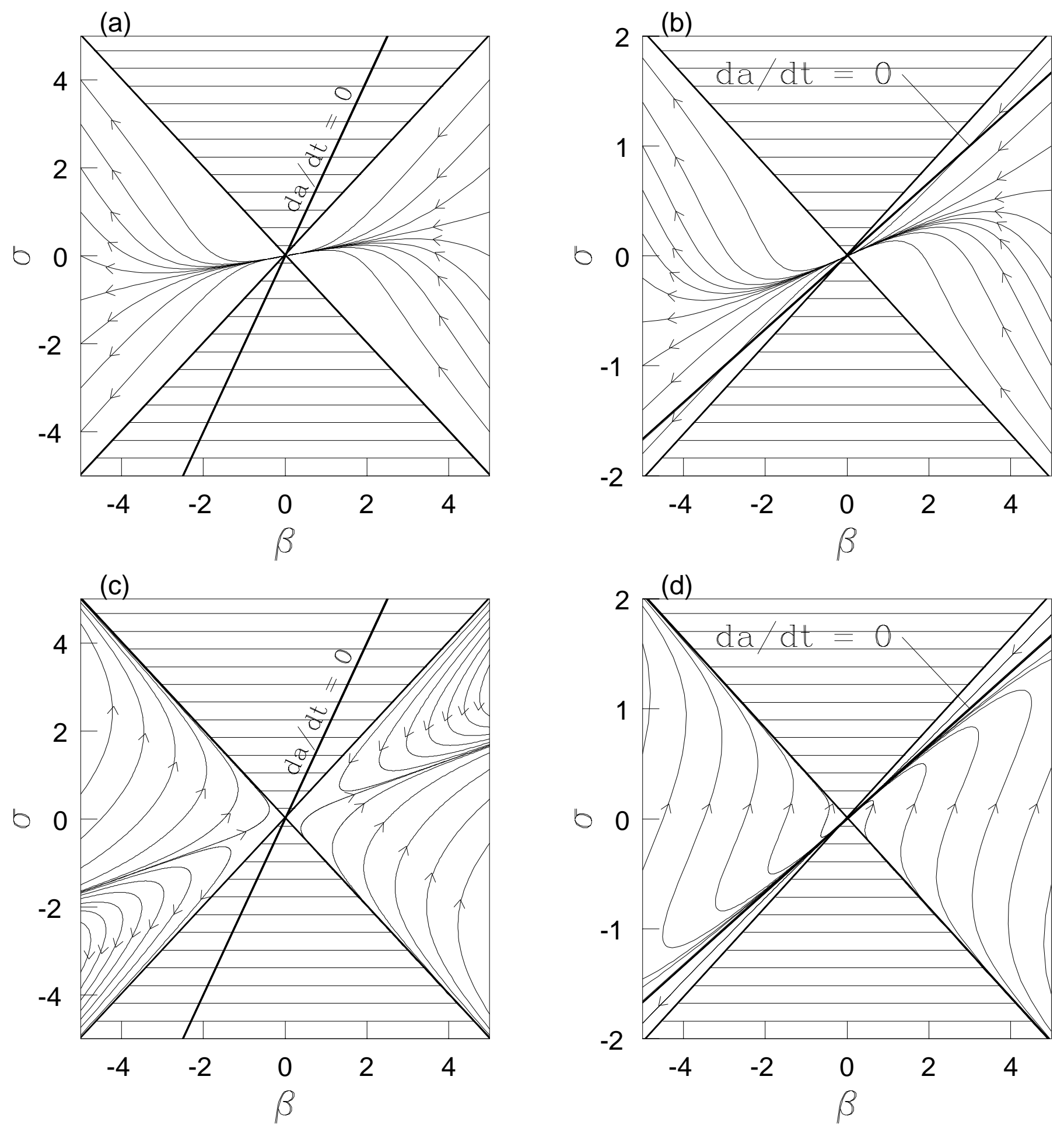

Fig. 1 

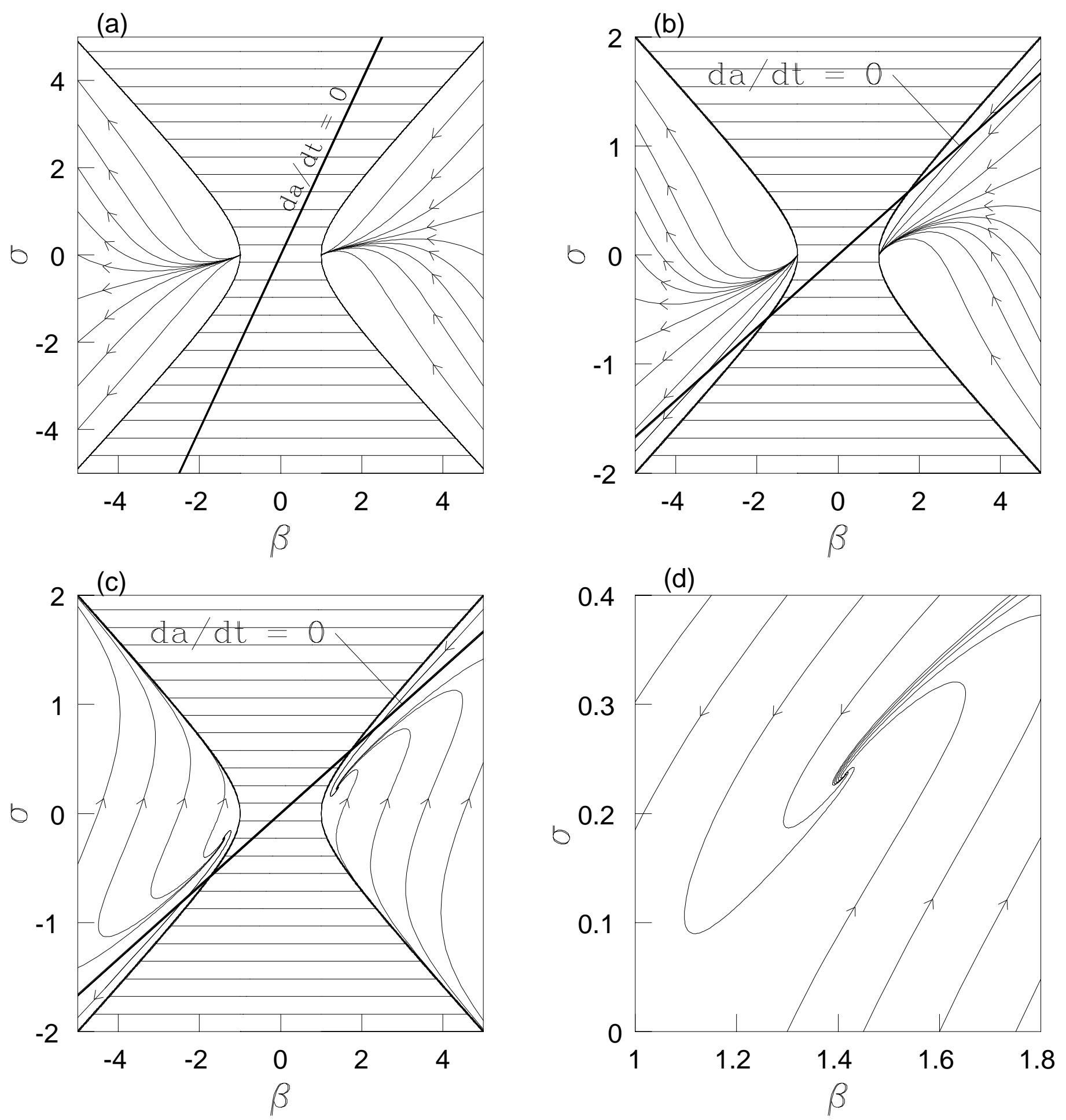

Fig. 2 
This figure "fig1-3.png" is available in "png" format from: http://arxiv.org/ps/gr-qc/9405016v2 
This figure "fig1-4.png" is available in "png" format from: http://arxiv.org/ps/gr-qc/9405016v2 


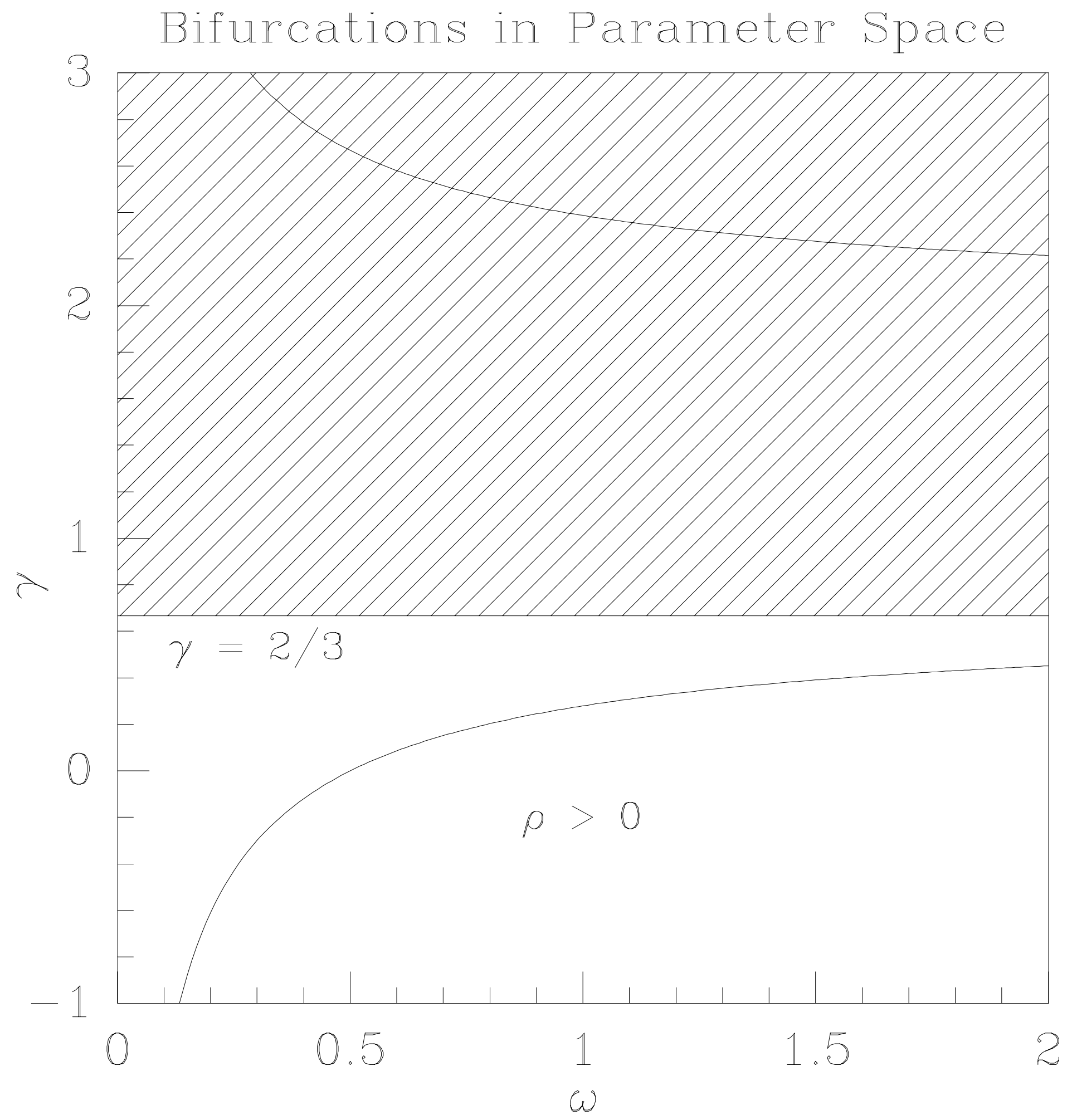

Fig. 3 

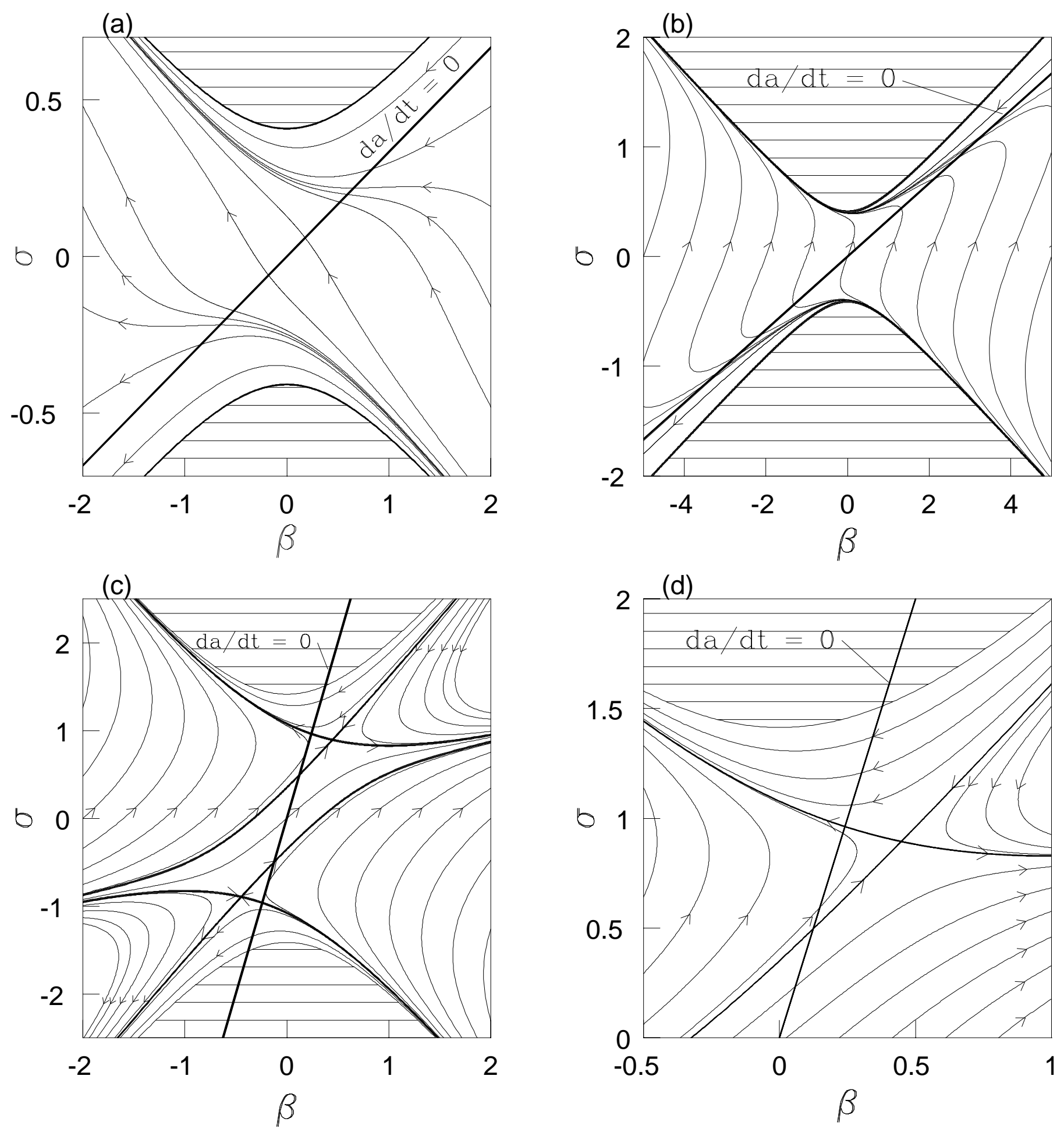

Fig. 4 